\title{
Non-mechanical beam steering: ways and means
}

\section{David M. Benton}

David M. Benton, "Non-mechanical beam steering: ways and means," Proc. SPIE 10797, Technologies for Optical Countermeasures XV, 107970H (9 October 2018); doi: 10.1117/12.2502758

SPIE. Event: SPIE Security + Defence, 2018, Berlin, Germany 


\title{
Non-Mechanical Beam Steering - Ways and Means
}

\author{
David M. Benton, \\ Aston Institute of Photonic Technologies, Aston University, Aston Triangle, Birmingham, UK \\ d.benton@aston.ac.uk
}

Keywords: Beam steering, non-mechanical beam steering, micromirrors.

\section{ABSTRACT}

One of the very useful aspects of a laser is its well-defined beam, delivering high intensity to a defined location. Directing that beam and specifying the location is generally done with adjustable mirrors. Directing the beam in time varying manner most often requires galvanometer scanning mirrors which translate in one dimension. These mirrors, though now a mature technology, are in general speed limited due to their inertia and can be heavy, power hungry and expensive. There are then benefits to be gained from non-mechanical means of beam steering particularly in terms of speed and weight. This paper gives an overview of methods employed to implement beam steering and then concentrates on methods that do not rely on independent phase control. The use of a micromirror array for 3-dimensional beam control will be presented with the pros and cons that this entails.

\section{INTRODUCTION}

The ability to steer a beam of light has been required for thousands of years. The ancient Egyptians used shiny metal mirrors to direct sunlight along tunnels to provide illumination for decorating underground tombs. That same principle is still in operation today. The beams tend to be generated by a laser but directing the beams is usually done using a mirror. For active control of the beam pointing, scanning galvanometer mirrors are used providing a one dimensional control. When large apertures are required the mass (or rather inertia) of the mirror limits the speed at which the beam can be redirected. It also influences the size, weight and power consumption of the directing system and generally such systems require a strong and stable platform. Mechanical steering can be

achieved with mirrors ${ }^{1}$, rotating prisms ${ }^{2}$ or piezo activation ${ }^{3}$. The concept of non-mechanical beam steering is therefore attractive as a means of overcoming some of the issues that arise from mechanical steering. Non-mechanical beam steering can be achieved through means such as acousto-optic deflection ${ }^{4}$, electro-optic deflection and the use of spatial light modulators ${ }^{5}$. In the current context it might be more accurate to refer to non-inertial beam steering where the mass of the system does not limit the response time, although other systems do not always respond at significantly faster rates.

\section{NON-MECHANICAL BEAM STEERING}

Non-mechanical beam steering is often implemented by controlling the phase structure of a beam using an array of phase control points or sources and is well summarised in a review by

\footnotetext{
Technologies for Optical Countermeasures XV, edited by David H. Titterton, Robert J. Grasso,

Mark A. Richardson, Proc, of SPIE Vol 10797, 107970H - C) 2018 SPIE

CCC code: $0277-786 X / 18 / \$ 18 \cdot$ doi: $10.1117 / 12.2502758$
} 
McManamon ${ }^{6}$. This paper is concerned more with devices that manipulate phase rather than control the phase in an absolute sense but will also mention interesting alternatives. One such interesting approach was taken by Zohrabi et $\mathrm{al}^{7}$ who used a series of tunable liquid lenses to implement beam steering. Steering in one dimension (1D) is accomplished by offsetting the optical axes of 2 tunable lenses and then controlling the focusing power of both lenses to focus the transmitted beam in a fixed focal plane. A diffuser is placed at this focal plane to act as a point source with known scatter angle, and a conventional lens system is used to generate the beam. With three lenses offset in orthogonal directions 2D beam steering can be achieved. With a fish eye lens system this can achieve large steering angles of around 70 degrees. However the system is extended in length $(>300 \mathrm{~mm})$ and its scanning rate will be limited by the response of the liquid lens.

Beam steering for use with indoor optical communications is being developed by Oh et al ${ }^{8}$. Their approach is to use fixed gratings to diffract the beam and control the diffraction angle by controlling the wavelength of the laser beam. This would give 1D beam steering. Beam steering in 2 dimensions was accomplished using a high output count array waveguide grating ${ }^{9}$, with the output fibres arranged as a 2D array. A collimating lens was used with the fibres at its focal plane and beam direction is decided by which fibre is emitting - which is in turn determined by the wavelength used. The concept as in the 2D case above, of beam steering by selection of spatially discrete sources located at the focal plane of a lens is a seemingly little used concept for beam steering. Toyoshima et $\mathrm{al}^{10}$ used an addressable $8 \times 8$ VCSEL array placed at the focal plane of a lens with a view to designing an optical communications system for satellite communication. Beam steering is achieved by selecting an appropriate emitter and the beams have a level of overlap to ensure no gaps in angular coverage within the field of view. A similar approach has been investigated by the author with a view to investigating handheld optical communications.

One of the more novel and interesting approaches to non-mechanical beam steering uses polarisation sensitive gratings (PG) to steer beams ${ }^{11}$ A PG consists of a grating structure built from switchable nematic liquid crystal (LC). A steering unit requires a polarisation defining waveplate followed by a PG. Light is steered into one of 3 possible directions - linearly polarised goes straight through, RCP sent to one order, LCP to the opposite. With 3 possibilities each stage multiplies the number of output angles. Thus a 3 stage device has 9 steering angles. They have demonstrated non mechanical beam steering with a 44 degree field of regard with 1.7 degree resolution, with over $78 \%$ optical throughput. This is a 1D system but a 2D steering system has also been demonstrated using a large aperture $(100 \mathrm{~mm})$ LC. A 4X expansion telescope was incorporated giving 64 degree $\mathrm{x} 64$ degree steering in steps of 1 degree.

Controllable 2D structures (arrays) can enable both steering and focusing of an output beam. Fresnel zone plates (FZP) displayed on a spatial light modulator (SLM ) are an attractive way of steering and focusing via non-mechanical means. Lindle et $\mathrm{al}^{5}$ used a LC SLM to render Fresnel zone plates. This is strictly a phase control approach as the phase at each pixel is controllable but is relevant here for its non-mechanical nature and its relation to what follows. 


\section{BEAM STEERING WITH A DIGITAL MICROMIRROR ARRAY}

The digital micro mirror device (DMD) is a type of SLM which uses a 2D array of tilting MEMS mirrors to control spatial light distributions and is a mature technology found within digital projectors for over 2 decades. One attempt at beam steering with a DMD used a conceptually similar approach to the spatial array of sources at the focal plane of a lens ${ }^{10}$. With off axis illumination, a DMD at the focal -plane of a lens means each mirror acts like a source and beams are steered by selecting appropriate mirrors in the array to reflect. This is however a very inefficient approach. Recent publications by Lindle and Watkin ${ }^{12}$, and Benton ${ }^{14}$ have demonstrated beam steering using FZP on DMDs. Whilst the DMD is not in the truest sense of the phrase a nonmechanical system, the response time of the mirrors is fast enough for it to be considered a noninertial limited system - mirror switching rates of $22 \mathrm{kHz}$ vs around $1 \mathrm{kHz}$ for LC. Although utilising an array the concept does not control phase, in this case it rather manipulates phase polarity (a spatial phase diode). The binary on-off nature of DMD pixels is very well suited to the use of binary FZP's. The use of a DMD offers some distinct benefits over other approaches, namely fast switching speed, robust technology base, wide spectral bandwidth and random access focusing - the ability to switch between one focused beam position and a completely different one in a single step - and multiple independent beam control. The main disadvantage of using DMDs is a poor efficiency caused by scattering and diffraction into multiple orders. Mirror sizes of $13.6 \mu \mathrm{m}$ lead to diffraction orders separated by 2.67 degrees using a wavelength of $635 \mathrm{~nm}$. The field of view is also limited due to the effective blaze angle of the device.

A schematic diagram of the system setup is shown in Fig.1. This is essentially the simplest system possible consisting of a collimated laser beam expanded sufficiently to cover the DMD, the DMD itself, a computer to control the DMD pattern and a screen to view the resulting beams. Three dimensional beam steering (horizontal, vertical and focus) is performed entirely by the DMD which has very little depth, having no other optics involved. Multiple diffraction orders are always seen in reflection from a DMD, they are the unavoidable consequence of using a 2D array. A focused diffraction spot can be seen at each diffraction order resulting from the FZP patterns. This can be useful in extending the field of view where the edge of one order meets the beginning of the next and continuity can be obtained. Because of the tilted mirrors the DMD is an off axis device and from the perspective of the focal point the FZP is squeezed into a series of ellipses. The results in different focal powers in horizontal and vertical directions and causes astigmatism to appear in the focus. This can be reduced by adjusting the FZP pattern to stretch in one direction and appear circular from the focus position. 


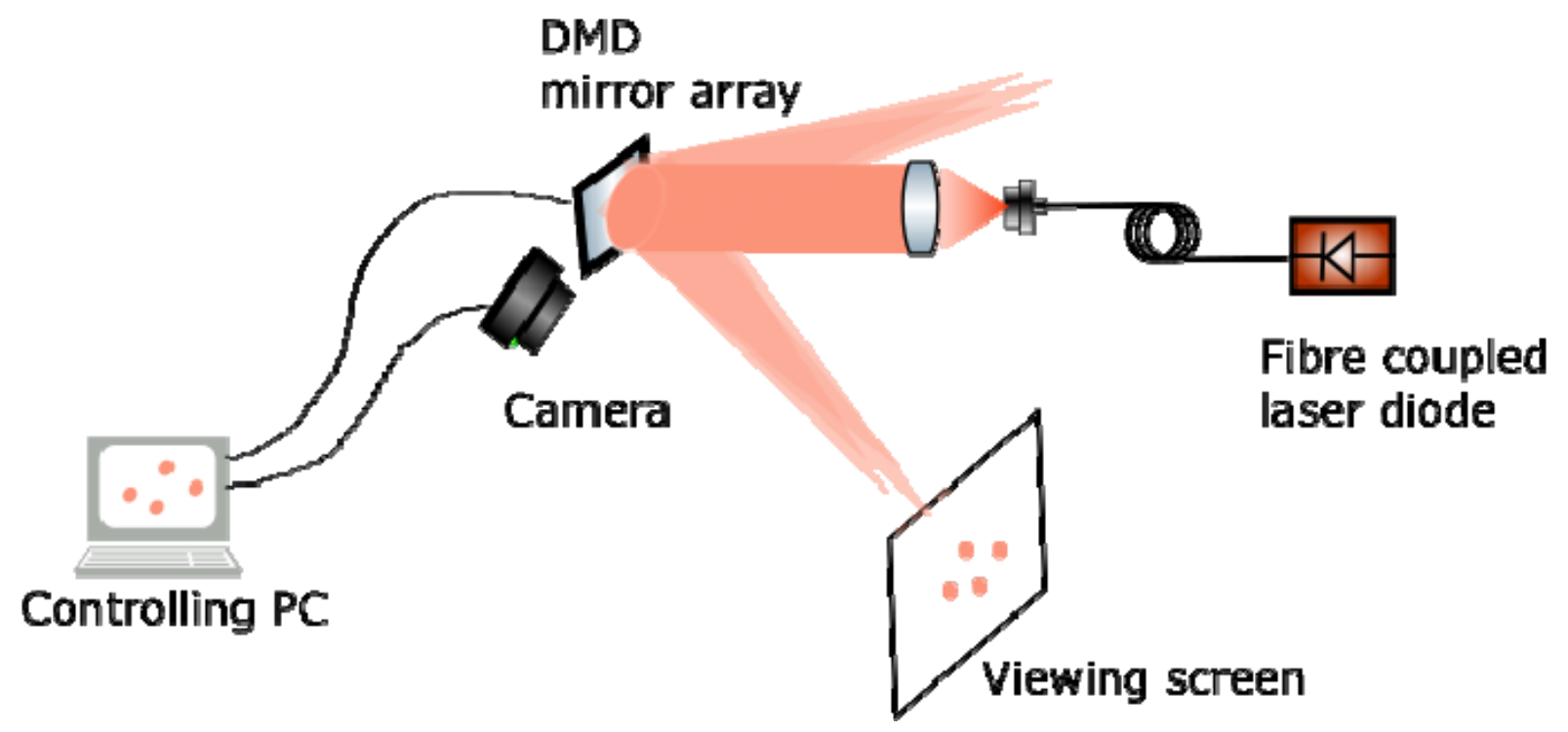

Figure 1. A schematic diagram of the DMD beam steering system.

A USB camera connected to the computer produces an image of the diffracted spots. This digital image is used to control the beam. Fig 2 shows a photograph of the computer screen displaying the camera image on the left and the actual beam spots on the right. Clicking a mouse pointer on the computer image moves the focused beam spots to that point by shifting laterally the FZP patterns. Fine control can be implemented as subpixel size shifts result in output beam shifts. The mouse control can manipulate the beam positions in real time at the camera rate of $30 \mathrm{fps}$. This demonstration was produced to show that a camera image can be used as the targeting source for beam steering, such that targets could be tagged, tracked and illuminated in a single system.

A separate software control was written for focus optimisation -suitable when the spot size on the screen as seen by the camera is more than a few pixels in extent. This used the random access nature of the DMD by examining the focal spot size at 3 widely spaced focal values, then using a polynomial fitting routine to home in by estimating the correct focus and repeating at a finer scale. Such a system could also act as a crude range finder.

By defining and overlaying multiple FZP patterns onto the DMD multiple beams can be generated within each order- see Figure 3 . These beams can be independently controlled such that they have separate direction and focus, without any additional equipment - still just a DMD. Inevitably there is a reduction in efficiency as light is shared between the beams and lost at the combination conditions determining each pixel state. Independent beams could be used in tracking multiple targets within a field of view or could be used for comms links to specific points within a field of view. 


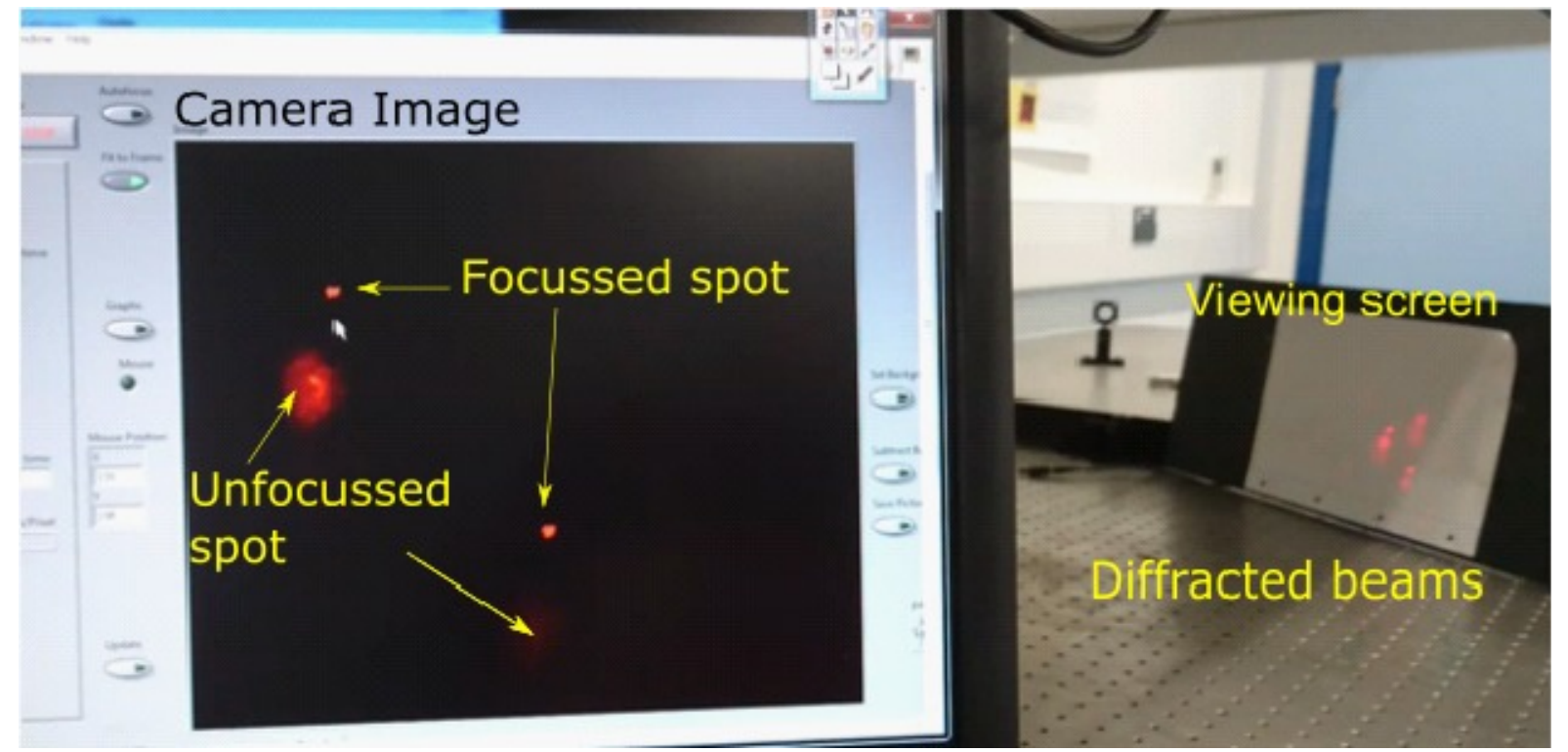

Figure 2. A snapshot of camera image of the beam spots displayed on a computer screen on the left hand side of the picture, with actual diffracted beam spots striking a screen on the right

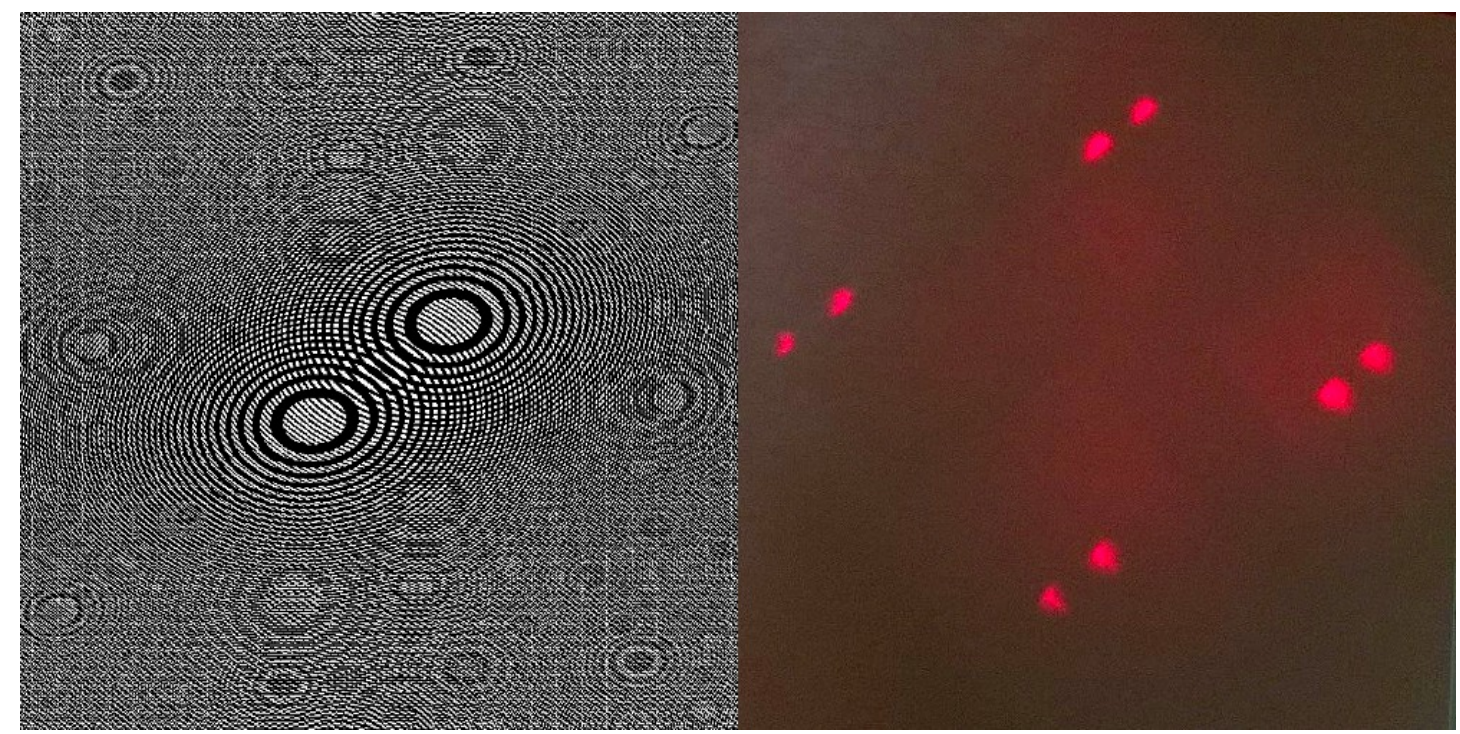

Figure 3. Two separate offset FZP patterns (left image) with corresponding separate focused beams seen in each diffraction order (right image).

\section{DISCUSSION}

When thinking about non-mechanical beam steering it would be wise to examine why there is a problem with mechanical beam steering and why, if that is the case it has not been displaced already. One issue is the perception that systems with "moving parts" are inherently unreliable and systems without movement should be used. This is likely to be a misconception as there are plenty of 
examples of reliable mechanical systems in use. Perhaps the principle issue is speed of response which might typically be $<1 \mathrm{kHz}$ and non-inertial limited systems could overcome this limitation. Accuracy of pointing requires a high degree of engineering and can significantly increase size, weight and cost of mechanical systems. Non-mechanical means are not immune from these same issues. For example a beam steering system used in the home would be unlikely to use a double galvanometer mirror system for reasons of size and power consumption, yet a more compact non mechanical system using, for example, tunable lasers and array waveguide gratings might be prohibitively expensive. Other systems using say tunable lenses might be equally bulky, whereas an SLM based system would not have sufficient field of regard. The use of non-mechanical beam steering is therefore very much application dependent. There must be specific advantages that non mechanical beam steering methods bring to particular applications - for example LC based system are not necessarily any faster than mechanical systems but would consume less power.

It is likely that hybrid systems will offer a more generic solution. In such a system low cost servo systems could point the beam in roughly the required direction with non-mechanical means providing the fine pointing control. In this way the engineering cost of high precision mechanical steering is removed but can help overcome issue of field of regard which limits some nonmechanical techniques. In addition the speed of response is provided by the non-mechanical system thus utilizing the best that both approaches have to offer.

\section{REFERENCES:}

1. Wehr and U. Lohr, "Airborne laser scanning - an introduction and overview," ISPRS J. Photogramm. Remote Sens. 54, 68-82 (1999).

2. B. D. Duncan, J. B. Philip, and S. Vassili, "Wide-angle achromatic prism beam steering for infrared countermeasure applications," Opt. Eng. 42, 1038-1047 (2003).

3. B. S. Kim, S. Gibson, and Tsu-Chin Tsao, "Adaptive control of a tilt mirror for laser beam steering," American Control Conference, Proceedings of the 2004. 4, 3417-3421 (2004).

4. S. R. Davis, G. Farca, S. D. Rommel, A. W. Martin, and M. H. Anderson, “Analog, Non-Mechanical Beam-Steerer with 80 Degree Field of Regard," Proc. SPIE 6971, 69710G (2008).

5. J. Lindle, A. Watnik, and V. Cassella, "Efficient multibeam large-angle nonmechanical laser beam steering from computer-generated holograms rendered on a liquid crystal spatial light modulator," Appl. Opt. 55, 4336-4341 (2016)

6. McManamon, P. F., Bos, P. J., Escuti, M. J., Heikenfeld, J., Serati, S., Xie, H., \& Watson, E. a. (2009). A review of phased array steering for narrow-band electrooptical systems. Proceedings of the IEEE, 97(6), 1078-1096. https://doi.org/10.1109/JPROC.2009.2017218

7. Zohrabi, M., Cormack, R. H., \& Gopinath, J. T. (2016). Wide-angle nonmechanical beam steering using liquid lenses. Optics Express, 24(21), 23798. http://doi.org/10.1364/OE.24.023798

8. Oh, C. W., Tangdiongga, E., \& Koonen, A. M. J. (2014). Steerable pencil beams for multi-Gbps indoor optical wireless communication, 39(18), 5427-5430.

9. Koonen, Ton, Gomez-Agis, Fausto, Mekonnen, Ketemaw Addis, Cao, Zizheng \& Tangdiongga, Eduward (2018). High-capacity optical wireless communication using 2-dimensional IR beam steering. Journal of Lightwave Technology, 2018.

10. Toyoshima, M., Miyashita, N., Takayama, Y., Kunimori, H., \& Kimura, S. (2009). System analysis of nonmechanical compact optical transceiver for wireless communications with a VCSEL array. Journal of Communications, 4, 582-587. http://doi.org/10.4304/jcm.4.8.582-587

11. Kim, J., Oh, C., Serati, S., \& Escuti, M. J. (2011). Wide-angle, nonmechanical beam steering with high throughput utilizing polarization gratings. Applied Optics, 50(17), 2636. https://doi.org/10.1364/AO.50.002636

12. Refai, H. H., Sluss, J. J., \& Tull, M. P. (2007). Digital micromirror device for optical scanning applications. Optical Engineering, 46(8), 85401. http://doi.org/10.1117/1.2768978 
13. Lindle, J. R., \& Watnik, A. T. (2018). Large angle nonmechanical laser beam steering at $4.6 \mu \mathrm{m}$ using a digital micromirror device. Optical Engineering, 57(02), 1. https://doi.org/10.1117/1.OE.57.2.027108

14. David M. Benton, "Multiple beam steering using dynamic zone plates on a micromirror array," Opt. Eng. 57(7), 073109 (2018), doi: 10.1117/1.OE.57.7.073109. 